\title{
Evaluating the degree of molecular contact between cellulose fiber surfaces using FRET microscopy
}

\author{
Georg Urstöger • Monica G. Simoes • Andreas Steinegger • Robert Schennach • \\ Ulrich Hirn (1)
}

Received: 15 April 2019/Accepted: 17 June 2019/Published online: 26 June 2019

(C) The Author(s) 2019

\begin{abstract}
The degree of molecular contact, i.e. the contact area on the nanometer scale, between paper fibers is crucial for the van-der-Waals and hydrogen bond adhesion between the fibers and thus for the fiber-fiber bond strength. We apply Förster resonance energy transfer (FRET) to investigate the degree of contact in the distance range of $1-10 \mathrm{~nm}$ between pulp fiber bonds and between thin films. The FRET system with DCCH and FTSC as fluorescence dyes has been validated for spectrophotometry and for local imaging with widefield microscopy, using pHema thin films. Bonding between thin films can be detected with this
\end{abstract}

system, however it has not been possible to achieve a significant FRET signal between bonded pulp fibers. Therefore, we conclude that in principle it is possible to quantify the degree of contact between two surfaces on the nanometer scale with the investigated FRET system. For further work on pulp fibers we recommend an exclusively surface active dyeing, as bulk dyeing massively deteriorates the signal to noise ratio which is likely the reason for the low FRET signal found in this work.

Electronic supplementary material The online version of this article (https://doi.org/10.1007/s10570-019-02575-x) contains supplementary material, which is available to authorized users.

G. Urstöger · M. G. Simoes · U. Hirn ( $\bowtie)$

Institute of Paper-, Pulp- and Fibre Technology TU Graz, Inffeldgasse 23, $8010 \mathrm{Graz}$, Austria

e-mail: ulrich.hirn@tugraz.at

\author{
A. Steinegger \\ Institute of Analytical Chemistry and Food Chemistry TU \\ Graz, Stremayrgasse 9/II, 8010 Graz, Austria \\ R. Schennach \\ Institute of Solid State Physics TU Graz, Petersgasse 16/2, \\ 8010 Graz, Austria \\ G. Urstöger · R. Schennach · U. Hirn \\ CD Laboratory for Fiber Swelling and Paper Performance, \\ Inffeldgasse 23, $8010 \mathrm{Graz}$, Austria
}




\section{Graphic Abstract}
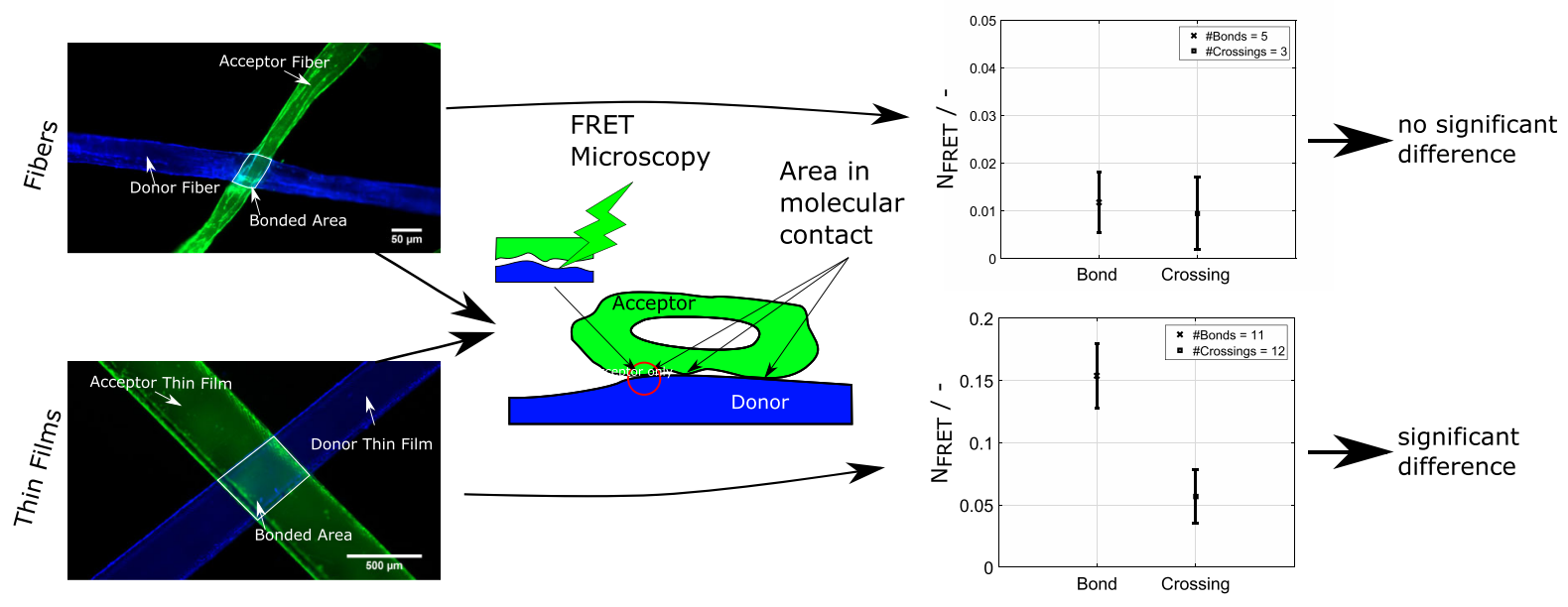

Keywords Adhesion - FRET - Contact mechanics · Förster resonance energy transfer · Cellulose fibers

\section{Introduction}

The strength of a paper sheet crucially depends on the strength of the adhesion between the bonded paper fibers. The fiber surfaces are not covalently bonded, still they exhibit adhesion between each other due to hydrogen bonding, van der Waals forces and Coulomb interaction (Lindström et al. 2005; Hirn and Schennach 2015). For hydrogen and van der Waals bonding the surfaces have to be closer than approximately 1 $\mathrm{nm}$. The contact area on the nanometer scale between the surfaces thus is in direct proportion to the adhesion between the surfaces (Persson 2003; Persson et al. 2004). This contact area on the nanometer scale is often termed "real contact area" or "area in molecular contact" (AIMC).

Measuring the area in molecular contact using imaging techniques is difficult as illustrated in Fig. 1. Regions that appear to have full contact at a given imaging resolution turn out to be only partially bonded when imaged using a higher imaging resolution (Yang et al. 2008). Hence the area in molecular contact between two surfaces appears smaller and smaller as the resolution of the imaging technique increases. In order to provide relevant results for adhesion the bonding area has to be evaluated on the sub-nanometer scale (Hirn and Schennach 2017).
A detailed review on the different approaches to measure area in molecular contact in fiber-fiber bonds can be found in the work of Hirn et al. (2013). Different imaging methods have been applied like polarized light microscopy (Kappel et al. 2010) or X-ray tomography (Sormunen et al. 2019). Both techniques are not able to resolve the bonding region in the nanometer range. Transmission electron microscopy (Asunaa and Steenberg 1958) is close to the relevant optical resolution, however the sampling area becomes so small that this method is also not suitable to provide quantitative results. Another approach to measure AIMC are adsorption based methods. The idea here is e.g. to dye fiber fiber bonds, then break the bonds and look which fraction of the bonding area is colored (Torgnysdotter et al. 2007), implying that the uncolored areas have previously been bonded. Similarly BET nitrogen absorption on paper sheets is used as an indicator for AIMC, e.g. Haselton (1954). The problem with these methods also becomes visible in Fig. 1. Unbonded areas can be surrounded by bonded regions, making them unaccessible for the adsorbent (dye or nitrogen). Adsorption based methods thus are also not able to quantify the AIMC.

Our approach to measure this area is Förster Resonance Energy Transfer Microscopy (FRET). The working principles of FRET are schematically explained in Fig. 2. It is based on the electromagnetic interaction (dipole-dipole interaction) between two dyes, a donor and an acceptor molecule (Förster 1948). When the donor dye molecule is excited two things 


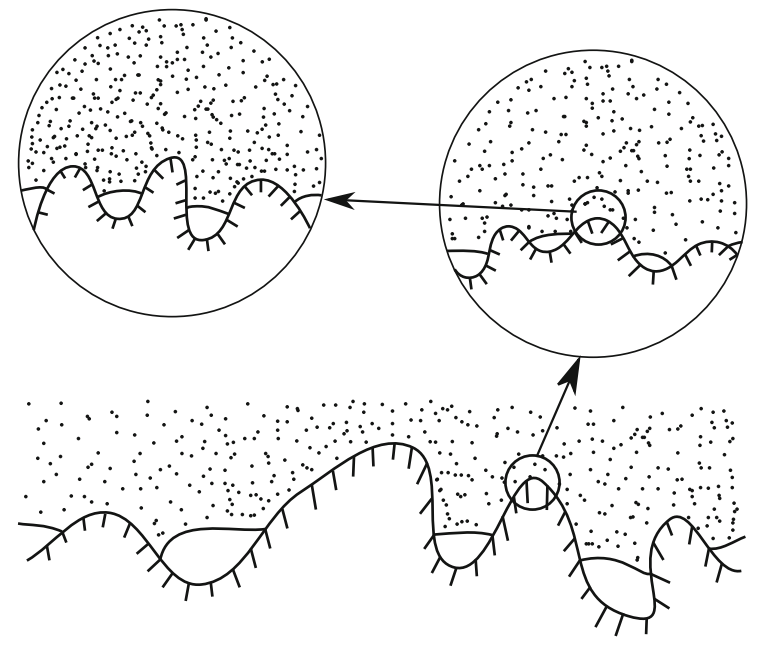

Fig. 1 Two surfaces in physical contact investigated under increasing magnification. As the magnification increases one can see that less area is in contact. Adapted from Persson et al. (2004)

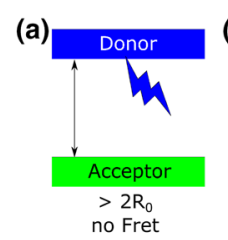

(b)

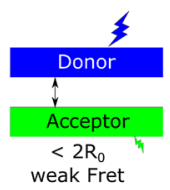

(c)

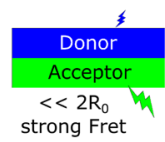

(d)

Mixture

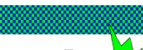

max. Fret th
Fig. 2 Explanation of the FRET effect. A FRET signal occurs if the distance between the dye molecules is smaller than $2 R_{0}$ (Förster Radius). In the cases a-c either surface is dyed with donor or acceptor dye, respectively. In this study the surfaces will be paper fibers (or polymer films). The donor dye is excited with light. As the donor and acceptor molecules between the surfaces come closer the acceptor fluorescence (FRET signal) increases and the donor fluorescence decreases. For a mixture of the dyes $\mathbf{d}$ a maximum FRET signal is observed

can happen. If an acceptor molecule is close the donor transfers its energy, by a non-radiative interaction, to the acceptor (Fig. 2c). The acceptor molecule is then emitting a photon, showing FRET fluorescence. The key point is that this FRET energy transfer, and the subsequent fluorescence of the acceptor molecule, only takes place if the donor and acceptor dye are close enough to each other, i.e. below $12 \mathrm{~nm}$ (Meer 2013). If there is no acceptor molecule close to the donor molecule, the donor emits a photon (Fig. 2a). The key prerequisite for FRET is that the fluorescence spectra of donor and acceptor are different but have a spectral overlap (see Fig. 4). By exciting the donor dye and recording the spectrum of the light emitted from the sample the amount of energy transferred to the acceptor dye can be measured. Also it should be mentioned here that additionally to the distance of the dyes also the orientation of the dipoles plays a role in the efficiency of the transfer.

For our work we are exploiting FRET to measure the degree of molecular contact between pulp fibers (and also between polymer thin films). The basic principle is sketched in Fig. 2a-c. There, one can see two blocks labelled donor and acceptor. These blocks stand for paper fibers where one fiber is dyed with the donor molecule and the other one with the acceptor molecule. A FRET signal is only possible if the distance between these two surfaces becomes sufficiently small. Hence in this work we were using FRET to investigate the degree of bonding between paper fibers and model thin films. The key reason why FRET is a suitable technique for measuring the AIMC is that it operates on the relevant size scale for van-der-Waals and hydrogen bonding, i.e. a signal is only obtained if the two surfaces have a distance below $12 \mathrm{~nm}$. If the surfaces are further apart than the critical distance $2 \mathrm{R}_{0}$ there is no FRET signal and only donor fluorescence (Fig. 2a). When the surfaces get closer there is an increasingly high acceptor fluorescence (i.e. FRET signal) with simultaneously decreasing donor fluorescence (b, c). When mixing the dyes together, e.g. in a polymer film, we obtain a very strong FRET signal, compare Fig. 2d. This configuration is used as a positive control as a mixture of molecules (with the same concentration as for the samples) provides an upper limit for the possible FRET intensity.

Literature reports qualitative work on FRET applied to fiber fiber bonds (Thomson et al. 2007, 2008a, b). In this work we are providing a quantitative investigation on FRET as a tool to measure the degree of nanoscale contact in fiber to fiber bonds. It will be demonstrated that with the employed technique it is not possible to measure a valid FRET signal between paper fibers. Nevertheless, it will also be shown that the proposed setup is a working FRET system, which will be demonstrated using pHema thin films. 


\section{Experimental}

Förster resonance energy transfer (FRET)

The following brief introduction to Förster Theory was taken from the book by B.W. Van der Meer (Meer 2013) and is based partly on the original papers by Förster. The efficiency of the energy transfer between donor and acceptor dye depends on the distance between the two molecules, and is in principle determined by the Förster Radius $\left(\mathrm{R}_{0}\right)$. The Förster Radius can be calculated for every combination of donor and acceptor molecules as can be seen in the supplementary information (Table $\mathrm{S} 1$ ). $\mathrm{R}_{0}$ is specific for every donor-acceptor pair in a particular system. The efficiency of the energy transfer $\left(\eta_{\text {eff }}\right)$ between donor and acceptor is given by Eq. 1:

$$
\eta_{\text {eff }}=\frac{1}{1+\left(\frac{r}{R_{0}}\right)^{6}}
$$

where $r$ stands for the distance between the donor and acceptor and $\mathrm{R}_{0}$ stands for the Förster radius of the donor-acceptor pair. Practically, the FRET efficiency can be measured by different methods which can be implemented in either microscopy setups or measured by spectrophotometry, both of which were used in this paper.

Regardless of the measurement method, a FRET process can be detected by different aspects of the effect. FRET changes the Donor emission intensity (Donor quenching), the acceptor emission intensity (Acceptor sensitation), the lifetime of donor fluorescence (Lifetime measurements) and the polarization of the light (Anisotropy measurements). Here we used two of the more common methods to measure FRET, namely donor quenching (DQ) and acceptor sensitation (AS). Donor quenching measures the decrease of the donor fluorescence due to FRET. Acceptor sensitation measures the increase of the acceptor fluorescence due to FRET. While DQ is an indication for FRET, one cannot be certain of it, as there are other mechanisms such as concentration quenching that can deactivate the excited donor. AS on the other hand provides conclusive evidence for FRET as the acceptor fluorescence can only be increased by some sort of energy transfer (Meer 2013). An excellent review about implementation and the pitfalls of the technique is provided by Broussard et al. (2013). One crucial part of using FRET that should be mentioned here is to meticulously check if the signal is indeed valid especially when spectroscopic data is not available. This can be done by using negative controls, dynamic FRET or applying more than one FRET technique.

Materials

The dyes 7-(diethylamino)coumarin-3-carbohydrazide (DCCH, Purity 95\%, CAS: 100343-98-4) and fluorescein-5-thiosemicarbazide (FTSC, Purity 99\%, CAS: 76863-28-0) were bought from Santacruz Biotechnology. Solvents N,N-dimethylformamide (DMF), Tetrahydrofuran (THF), and Acetonitril were purchased from VWR. All chemicals were used without further purification. Poly(2-hydroxyethyl methacrylate) (pHEMA) for manufacturing of thin films was purchased from Sigma Aldrich. The model films were deposited on PET foils. The pulp used was an unbleached softwood Kraft pulp from industrial production.

\section{Sample preparation}

For investigating pulp fibers [bleached Kraft wood, ratio spruce/pine (90/10)], the samples were prepared as described by Thomson et al. (2007). Fibers were dyed in $15 \mathrm{~mL}$ of a solution of either dye in DMF (1 $\mathrm{mmol} / \mathrm{L}$ and $0.2 \mathrm{mmol} / \mathrm{L}$ ) overnight at a $\mathrm{pH}$ of 5 , adjusted by adding $\mathrm{HCl}$. The dyes form a metastable bond with the reducing end of the cellulose molecule and by adding $\mathrm{HCl}$ this equilibrium is shifted towards the meta-stable bond species side. After the dyeing, sodium borohydrate $\mathrm{NaBH}_{4}(0.02 \mathrm{mmol}$ $\mathrm{NaBH}_{4}$ per $0.5 \mathrm{~g}$ fibers) was added immediately to the solution and allowed to react for $1 \mathrm{~h}$. The sodium borohydrate leads to a reductive amination of the dyes with the cellulose which results in a covalent bond between the reagents. After this reductive amination the samples were first washed in DMF and again washed by Soxhlet extraction with acetonitrile for at least $4 \mathrm{~h}$ to remove any non-covalently bonded dye. Subsequently the dyed fibers were stored in an aqueous pH 9 buffer solution using $0.4 \mathrm{~g} / \mathrm{L}$ borax. The fiber bonds were prepared by crossing one DCCH dyed and one FTSC dyed fiber in a droplet of water (pH 9) and subsequently drying them in a Rapid Köthen sheet former. For microscopy the fiber bonds were then transferred to microscopy slides. Fiber 
Crossings (negative control for FRET) were prepared by simply crossing two fibers and covering them with a microscopy cover slip.

The model films were prepared by doctorblading $500 \mu \mathrm{L}$ of a $10 \%$ pHema in a $95 / 5 \mathrm{EtOH} / \mathrm{H} 2 \mathrm{O}$ solution mixed with dyes dissolved in THF onto PET foils. This resulted in an approx. $1.5 \mu \mathrm{m}$ thick film. To ensure alkaline conditions a volume of $12 \mu \mathrm{L}$ triethylamine was added to the solution. The PET foils were cleaned with acetone before the doctorblading. FRET was investigated in two different model systems. In the first trial (Mixture sample) the dyes were added to the film as follows. Donor in pHema, Acceptor in pHema and Donor + Acceptor in pHema. The interaction of molecules within this film was investigated. In a second step (Bonds and Crossings) two films (Donor in pHema, Acceptor in pHema) were prepared, stacked together and left at 1 bar pressure at $93{ }^{\circ} \mathrm{C}$ for $3 \mathrm{~h}$ to form contact. The concentrations and applied measurement methods for the samples can be seen in Table 1.

\section{FRET from spectrophotometry}

Fluorescence spectra were recorded on a RF-5301PC, spectrofluorophotometer from Shimadzu. FRET experiments were performed with concentrations of molecules seen in Table 1. Also care was taken to minimize the exposure of any of the samples to ambient light to avoid photo bleaching. The model films were on the one hand investigated using this fluorometry setup and on the other hand they were investigated using a wide field microscope with the filter sets seen in Table 2. The spectra of the pHema films were recorded using the setup seen in Fig. 3. The samples were investigated under an angle that avoids the detection of the directly reflected light and only measures the fluorescence coming from the sample.

The efficiency of the FRET energy transfer is calculated from the measured spectra and is being explained in Fig. 4. Due to the energy transfer the donor emission is quenched $\left(\mathrm{I}_{D A}\right)$ with respect to the normal emission of the donor $\left(\mathrm{I}_{D}\right)$. The energy not emitted by the donor is then transferred to the acceptor and increases (or sensitizes) the emission $\left(\mathrm{I}_{A D}\right)$ compared to the normal acceptor sensitation $\left(\mathrm{I}_{A}\right)$. The spectral overlap seen in Fig. 4 is a necessary requirement for FRET and determines to a large extent the distance range of the effect as can be seen in Eq. 2 .

$$
\mathrm{R}_{0}=\left(\frac{9 \ln (10)}{128 \pi^{5} N_{A}}\left(k^{2} n^{-4} Q_{D} J\right)\right)^{1 / 6}
$$

$\mathrm{R}_{0}$ is the Förster radius, $\mathrm{k}$ is the orientation factor, $\mathrm{n}$ the refractive index, $\left(\mathrm{Q}_{D}\right)$ the quantum yield of the donor and $\mathbf{J}$ the overlap integral. The Förster radius determines the distance of the energy transfer as it is known

Table 1 Concentrations and parameters for the FRET measurements

\begin{tabular}{lllll}
\hline Sample & Conc. donor $(\mathrm{mmol} / \mathrm{L})$ & Conc. acceptor $(\mathrm{mmol} / \mathrm{L})$ & FRET investigation & Instrument \\
\hline pHema film & 3 & 3 & Mixture films & SP + WM \\
pHema bond/crossing & 3 & 3 & Between films & SP + WM \\
Fiber bonds & 0.2 & 0.2 & Between fibers & WM \\
Fiber crossings & 0.2 & 0.2 & Between Fibers & WM \\
\hline
\end{tabular}

$W M$ wide field microscopy, $S P$ spectroscopy

Table 2 Filter sets used for the Fluorescence Microscopy

\begin{tabular}{llll}
\hline Filter set & Excitation $(\mathrm{nm})$ & Dichroic mirror $(\mathrm{nm})$ & Emission \\
\hline Donor (D) & $436 \pm 10$ & 455 long pass & $480 \pm 20$ \\
Acceptor (A) & $500 \pm 10$ & 515 long pass & 520 1. pass \\
FRET (F) & $436 \pm 10$ & 515 long pass & 520 1. pass \\
\hline
\end{tabular}




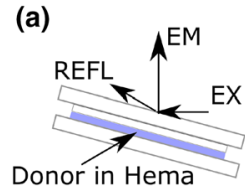

(b)

(c)

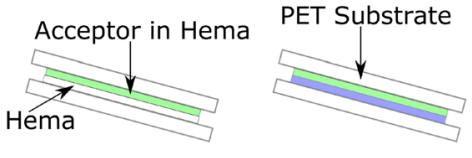

Fig. 3 Measurement setup of the pHema films in the fluorometry. In a the setup is explained in more detail. It was chosen such as that the reflected (REFL) beam following the excitation (EX) of the sample does not hit the detector and only the fluorescence signal emitted (EM) directly from the sample will be detected. a-c The Donor, Acceptor and Donor-Acceptor samples as they were investigated

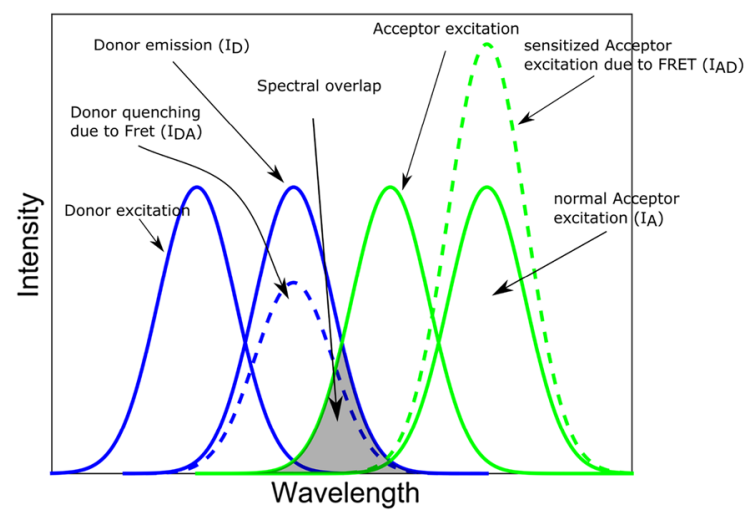

Fig. 4 Excitation and Emission spectra of a generic donoracceptor pair. FRET affects the donor emission intensity (donor quenching) and the acceptor emission intensity (acceptor sensitation). Also the spectral overlap of the donor emission and the acceptor excitation spectrum is shown.

that quantification of FRET is only possible within distances ranging from $\left(\frac{1}{2}-2\right) \mathrm{R}_{0}$ as also depicted in Fig. 2. A detailed description on how to determine the Förster radius can be found in FRET-Förster Resonance Energy Transfer by Medintz and Hildebrandt (Meer 2013).

The fluorescence emission spectra of DCCH and FTSC overlap to a certain extent as seen in Fig. 7. Therefore, to get an accurate measurement of the FRET efficiency the spectra need to be spectrally unmixed. To do this in the spectrometer we fitted the emission peaks and used the area under the peaks as the intensity of the fitted signal. Practically this was done by fitting Gaussian peaks to the recorded emission spectra of a pure donor sample (resulted in $\mathrm{I}_{D}$ ), a pure acceptor sample (resulted in $\mathrm{I}_{A}$ ) and a sample of interest (resulted in $\mathrm{I}_{D A}$ and $\mathrm{I}_{A D}$ ) such as in Fig. 11. The full fitting parameters can be found in the supplementary information (Figure S3 and Table S2). The resulting intensity information was used with Eqs. 3 and 4 to get the FRET efficiency. For the donor quenching (Eq. 3) and acceptor sensitation (Eq. 4) the following equations were used.

$$
\begin{aligned}
& \eta_{F R E T}=1-\frac{I_{D A}}{I_{D}} \\
& \eta_{F R E T}=\left(\frac{I_{A D}}{I_{A}}-1\right) \frac{\epsilon_{A}}{\epsilon_{D}}
\end{aligned}
$$

$\epsilon_{A}$ and $\epsilon_{D}$ are the extinction coefficients of the acceptor and donor at the excitation wavelength (Meer 2013; Clegg 1992, 1995). The ratio of $\frac{\epsilon_{A}}{\epsilon_{D}}$ was determined to be 0.02 and can be found in the supplementary information (Table S1). Acceptor sensitation and donor emission are sketched in Fig. 4.

\section{FRET from optical microscopy}

The paper fibers were investigated using a wide field microscopy (WM) setup equipped with the filter sets seen in Table 2. The WM was operated with a $50 \mathrm{~W}$ halogen lamp and a CMOS detector from QI Imaging (Optimos).

Both the intensity of the lamp and the detector sensitivity show a dependency of the wavelength and were corrected by calculating correction factors from the lamps emission spectrum folded with the excitation filters and the extinction coefficient and the detector sensitivity folded with the emission filters and the emission spectra. These correction factors were applied to the recorded images to get a true representation of the measured intensity.

A schematic of the dyed fibers and pHema films can be seen in Fig. 5. The images are false colored to emphasize the differences in the fibers/films. The samples were investigated using a $400 \times$ magnification
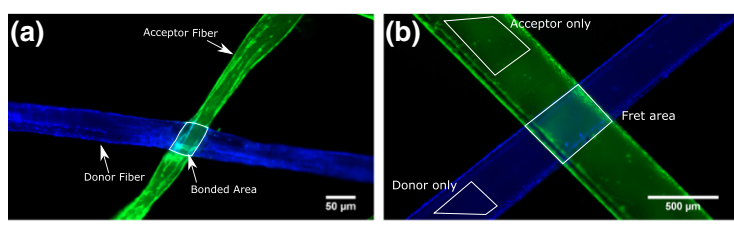

Fig. 5 False color images of a a fiber bond and $\mathbf{b}$ a pHema crossing measured in a wide field fluorescence microscope. In b also typical regions of interest for the Xia algorithms are shown. The regions are chosen to minimize scattered light from the other fiber/film 
in the case of fibers and $50 \times$ magnification for the case of films. To minimize background noise, the microscope was used in a box surrounded by black fabric.

When using the microscope one trades the spectral for spatial information and one loses the advantage to apply this simple fitting method described above. Here, a sophisticated algorithm that results in a fully corrected measure of the FRET efficiency has been developed by Gordon et al. (1998). The method makes use of images recorded with the three different filter sets from Table 2 of three different samples. Usually this would result in a total of nine images but with the arrangement seen in Fig. 5 it is possible to get the same information from only three images instead as in every image there is automatically a pure donor, a pure acceptor and the FRET area included. For a detailed description of the algorithm please refer to the original paper (Gordon et al. 1998). In brief this method calculates the FRET intensity corrected for all possible spectral bleed-through scenarios according to the following equations.

$$
\begin{aligned}
& \overline{A f a}=\frac{A f-\left(\frac{A d}{F d}\right) F f}{1-\left(\frac{F a}{A a}\right)\left(\frac{A d}{F d}\right)} \\
& F R E T 1=\frac{\left(F f-\left(\frac{F d}{D d}\right) D f-\overline{A f a}\left[\left(\frac{F a}{A a}\right)-\left(\frac{F d}{D d}\right)\left(\frac{D a}{A a}\right)\right]\right)}{G\left[1-\left(\frac{D a}{F a}\right)\left(\frac{F d}{D d}\right)\right]}
\end{aligned}
$$

$$
\overline{D f d}=D f+F R E T 1\left[1-G\left(\frac{D a}{A a}\right)\right]-\overline{A f a}\left(\frac{D a}{A a}\right)
$$

$$
F R E T N=\frac{F R E T 1}{\overline{A f a} * \overline{D f d}}
$$

The equations consist of variables with 2 letters. The first letter stands for the used filter set as seen in Table 2, the second letter for the investigated sample ( $d=$ donor only, $\mathrm{a}=$ acceptor only, $\mathrm{f}=$ FRET area). E.g. Af therefore stands for the FRET region (bonded area) investigated with the acceptor filter set. The variables represent the measured light intensities from the aforementioned microscope images recorded as 16 bit gray values. $\overline{A f a}$ refers to the acceptor signal that would have been if no donor were present and therefore no FRET occurred. Similarly, $\overline{D f d}$ refers to the donor signal that would have been if no acceptor were present and therefore no FRET occurred.
Regions of interest were chosen in the images as depicted in Fig. 5b. The evaluation was performed pixelwise. $G$ is a factor relating the loss of the donor signal to the increase of the acceptor signal (see Supplementary information Table S3). In this work we used the slightly different algorithm by Xia et al. which differs solely in the normalization of the $\mathrm{N}_{\text {FRET }}$ value but is otherwise equivalent to the Gordon algorithm (Xia and Liu 2001).

$$
N_{F R E T}=\frac{F R E T 1}{\sqrt{\overline{A f a} * \overline{D f d}}}[-]
$$

For being able to reproduce the area in molecular contact a method was developed to always choose the appropriate area. The method consisted of manually drawing an ROI (region of interest) capturing the "optically-bonded" area in Fig. 6. Subsequently we applied image erosion to avoid the edges and thus obtain the Eroded ROI (Fig. 6, bottom images) which was then used for evaluation of FRET intensity. Avoiding the edges was necessary because regions of extreme intensity appear to give a false positive FRET signal. Additionally, false positives were also detected on areas that are impossible to exhibit FRET such as the area seen in Fig. 6a that is not in the bonded region.

\section{Other FRET experiments}

It should be mentioned here that other FRET experiments were also employed. A confocal laser scanning microscope (CLSM, Leica TCS SPE) with a photomultiplier tube from Hamamatsu was used to investigate acceptor photobleaching and sensitized emission of the acceptor in fiber-fiber bonds. The idea was that due to the superior setup a better signal to noise ratio will be achieved.

\section{Results and discussion}

Excitation (EX) and emission (EM) spectra

To check that the combination of dyes can be used as a FRET system on the chosen substrates and the chosen $\mathrm{pH}$ value the excitation and emission spectra of the dyes on both substrates at a $\mathrm{pH}$ of 9 were 

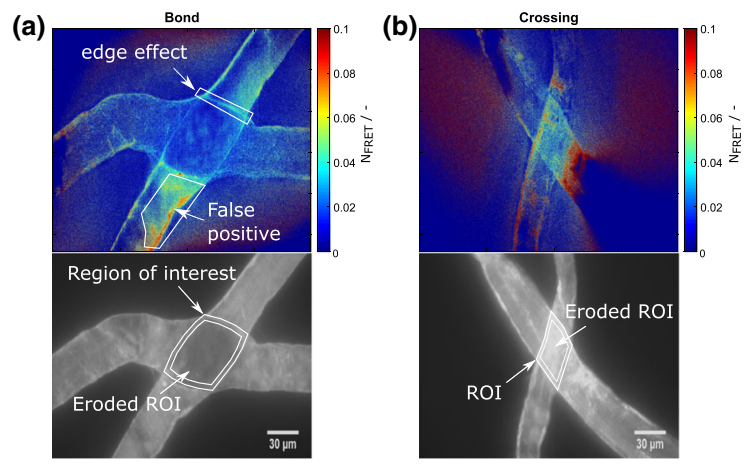

Fig. 6 Demonstration of the FRET analysis of a a fiber bond and $\mathbf{b}$ a fiber crossing. In a two typical problems with the analysis can be seen. First a high FRET signal was often detected on the edges of both, the bonded area and also the fibers. Secondly, a strong false positive FRET signal can be seen on an area that is clearly not possible to exhibit FRET. In the microscopy image the erosion technique used for analysis can be seen

investigated. An optimal setup would have a significant spectral overlap between the donor emission spectrum and the acceptor excitation spectrum (Overlap Integral, necessary condition for FRET) while having as little spectral bleed-through as possible. The resulting spectra can be seen in Fig. 7. As can be seen the donor emission and the acceptor excitation spectra show significant overlap. However also there is partial spectral overlap of the donor emission and the acceptor emission and vice versa. Also the acceptor excitation spectrum overlaps with the donor excitation spectrum. This spectral bleed through in the fluorescence microscopy is the reason for the necessity of the Xia algorithm in the optical microscopy and the spectral unmixing in the spectrophotometry to correct the signal. When comparing the dyes in the different substrates one can see some changes. First one can see that the excitation spectra of both dyes change at approx. $400 \mathrm{~nm}$ when comparing the fibers and
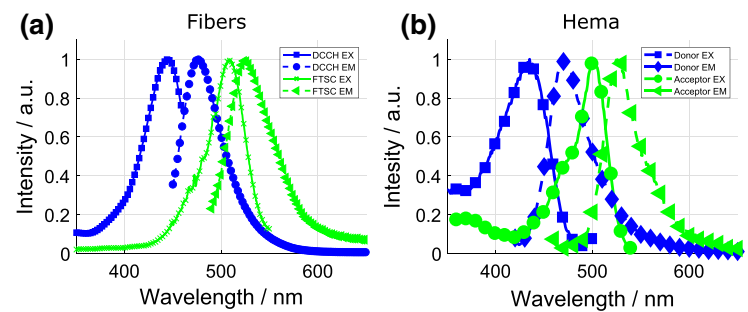

Fig. 7 Excitation and emission spectra of DCCH and FTSC on a paper fibers and $\mathbf{b}$ in pHema. Spectra are normalized to their peak value
pHema. Additionally, the FTSC spectra slightly shift in wavelength. These changes are attributed to the covalent linking and therefore chemical modification of the dye molecules. That the covalent bonding has an effect on the dyes can also be seen in the quantum yield reported in the supplementary information (Table S1). The changes are low enough to be able to compare the two systems with each other.

\section{Investigation of FRET between paper fibers}

Paper fiber bonds and crossings were investigated to see if it is possible to measure the area in molecular contact between two paper fibers using FRET fluorescence microscopy. When investigating fiber bonds one would expect a FRET signal between the fiber surfaces because the two surfaces and therefore also the dye molecules come very close. To check the plausibility of the signal, unbonded fiber crossings were investigated as a reference. As can be seen in Fig. 8 for both dye concentrations $(0.2 \mathrm{mmol} / \mathrm{L}$ and 1 $\mathrm{mmol} / \mathrm{L}$ ) it is not possible to detect a significant difference between the bond and the crossing. For illustration also refer to Fig. $6 \mathrm{~b}$ where a considerable false FRET signal can be seen in the overlapping (but unbonded) regions of the fiber crossing. A possible concentration dependence of the FRET signal was also investigated but did not show any improvement. These results show that it is not possible to measure the interaction of two paper fiber surfaces by using the employed method.

One reason for not being able to detect a difference in the fibers could be a low signal to noise ratio. If the dyes stay only at the surface of the fibers the signals can only originate from a thin layer of interaction. If, however the complete bulk is dyed it would likely lead
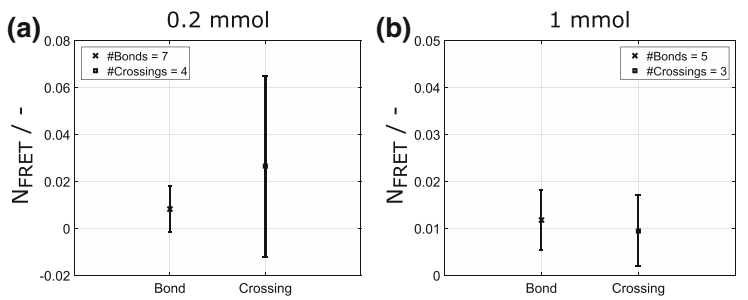

Fig. 8 Results from the analysis of 12 bonds and 7 not bonded crossings. a The fibers were dyed with a concentration of $0.2 \mathrm{mmol} / \mathrm{L}$. b The concentration was increased to $1 \mathrm{mmol} / \mathrm{L}$. In both cases the fiber bonds do not show a higher FRET signal than the crossings. Error bars are $95 \%$ confidence limits 

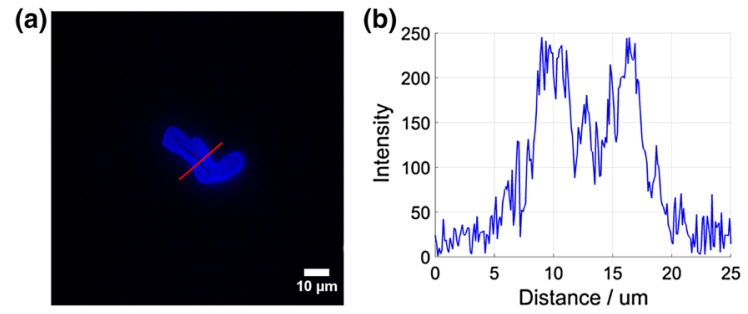

Fig. 9 a Cross section of a dyed paper fiber in false color. b Intensity Profile of the Line indicated in $\mathbf{a}$. The cross section as well as the profile shows that the complete bulk of the fiber was dyed

to a disadvantageous signal to noise ratio. To investigate this the cross sections of the fibers were looked at by using a confocal laser scanning microscope. The results of this investigation seen in Fig. 9 show that the complete bulk of the paper fibers with a thickness of up to $10 \mu \mathrm{m}$ was dyed by the employed method. This continuous dyeing of a $10 \mu \mathrm{m}$ thick bulk will lead to a very low signal to noise ratio as the following argumentation shows. The energy transfer due to FRET is only possible up to 2 times the Förster distance. In our case the Förster distance was measured to be about $6 \mathrm{~nm}$. (supplementary information Table S1) This means that a minimum distance of $12 \mathrm{~nm}$ is needed to get a FRET signal. In other words this means that when our paper fibers are bonded a thickness layer of approx. $10 \mathrm{~nm}$ is interacting. Comparing this to a fiber thickness of up to $10 \mu \mathrm{m}$ this results in a signal to noise ratio of 1:1000. Therefore, if there is even only little spectral bleedthrough it will strongly interfere with the FRET signal. This argumentation shows that if one wants to measure a FRET signal between two surfaces it is necessary to maximize the signal to noise ratio either by using thinner substrates or by confining the dyes to the region of interaction.

\section{Dynamic FRET experiments}

Another way to see whether a FRET experiment is successful or not is to investigate the dynamic development of the FRET signal. In our case we tried to replicate the experiments done by Thomson et al. (2007) in which the drying of a fiber bond was monitored. During paper drying the capillary forces of the retracting water are pressing the fiber surfaces together, thus creating the molecular contact area that
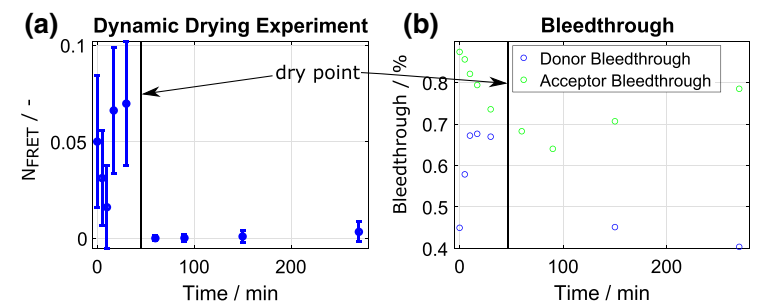

Fig. 10 In a one can see the development of the $\mathrm{N}_{F R E T}$ value as determined with the Xia algorithm. Initially it decreases before it increases again and subsequently finally drops to almost zero. In $\mathbf{b}$ one can see the donor and acceptor bleed-through as it develops during drying

facilitates adhesion between the fiber surfaces (Persson et al. 2012). In this investigation 6 fiber bonds were investigated that all show a similar behavior as to the one seen in Fig. 10. The collected measurements can be found in the supplementary information (Figure S2). As can be seen in Fig. 10a the FRET intensity is largest below the "dry point". The dry point is the time at which no water could be detected in the microscopy images. After this point the $\mathrm{N}_{\text {FRET }}$ drops to almost zero and stays there. Hence, we were observing the opposite of what one would expect. In the wet state where the fibers are certainly not bonded the FRET signal is the strongest and decreases to zero during the drying where the signal should increase. In Fig. 10b one can see that the spectroscopic bleedthrough between the filter sets changes over the drying period which is a partial reason for the unexpected development of the $\mathrm{N}_{\text {FRET }}$ characteristic. Since the bleed-through values are a measure of the spectroscopic properties of the dyes this means that as the water is reduced the excitation and emission spectra of the dyes bound to the fibers appear to be changing. In other words, this means that during drying the properties of the dyes are changing as well which makes a comparable analysis between measurements difficult. The reason for this change could be the change in $\mathrm{pH}$ when the amount of water is going down and thus the chemicals concentration is changing. Another reason for the trend could be the worse signal to noise ratio when investigating structures in water due to the stronger scattering of the light.

In summary both experiments aiming to validate the FRET system at hand did not show an increase in FRET signal between bonded and unbonded fibers. Thus the investigated FRET technique seems not suited to provide qualitative or quantitative 
information on the molecular contact area related to the adhesion between the fibers. The possible reasons for this is firstly and most likely the poor signal to noise ratio but also reflection and scattering of light on the fibers proves to be a difficult obstacle to overcome as it apparently leads to artifacts in the FRET signal.

FRET in pHema model films using spectroscopy

Our next step was to see whether it is possible to measure a FRET signal using the two dyes mixed together within one film. This measurement serves as a proof of concept that the employed dye system is in fact applicable as a FRET pair. The results are

(a)

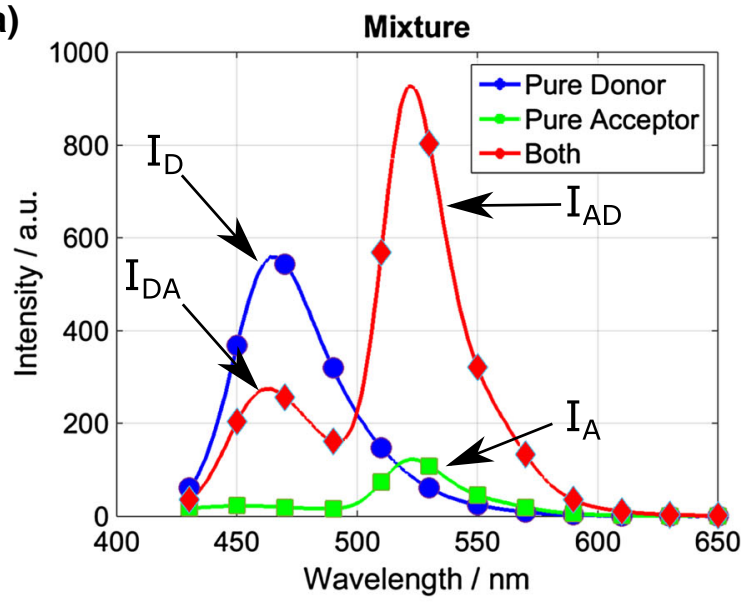

(c)

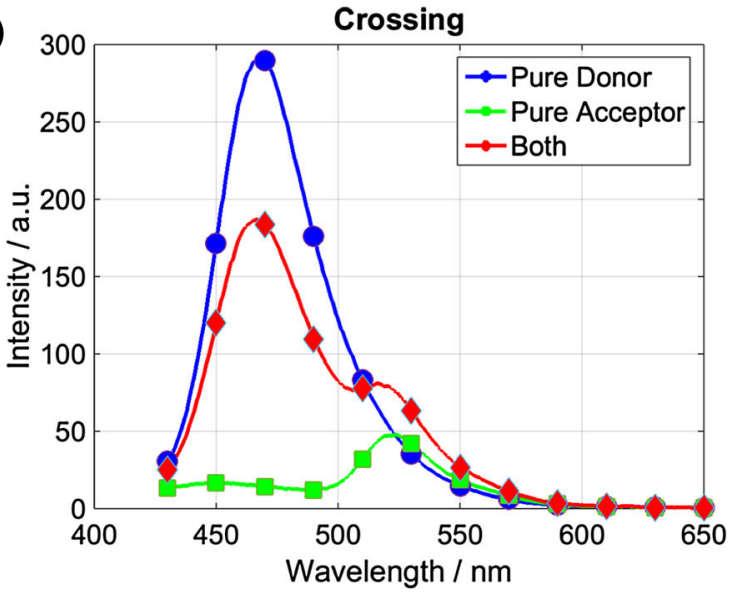

Fig. 11 Comparison of the FRET measurements between 3 types of systems in the spectrofluorometer. a Strong FRET signal stemming from the interaction of dyes mixed together within one film. b Weaker FRET signal coming from two separate pHema films bonded (bond) together. c No FRET presented in Fig. 11a. There one can see the clear behavior of a FRET system. In the presence of the acceptor the donor decreases (donor quenching $\mathrm{I}_{D}$ to $\mathrm{I}_{D A}$ ) in magnitude and transfers its energy to the acceptor (acceptor sensitation $\mathrm{I}_{A}$ to $\mathrm{I}_{A D}$ ) which shows a strong increase. The curves in Fig. 11 were fitted with Gaussian peaks and the values for $\mathrm{I}_{D}, \mathrm{I}_{A}, \mathrm{I}_{D A}$ and $\mathrm{I}_{A D}$ were extracted and used for the calculation of the FRET efficiencies, Eqs. 3 and 4, results are seen in Table 3, line 1. As presented a clear FRET response was measured using the spectrofluorometry.

In another experiment we wanted to see if we can measure a FRET interaction between two initially separated films as depicted in Fig. 2c. To achieve this,
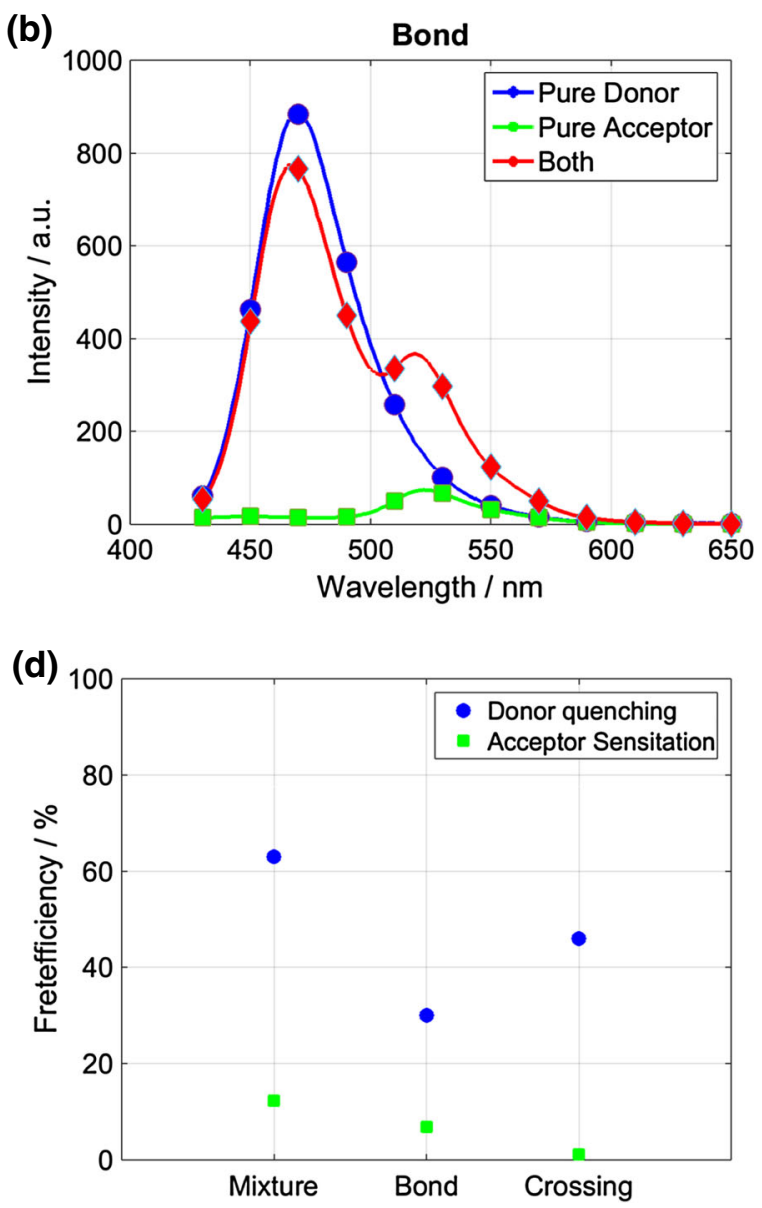

signal in the case of unbonded (crossing). d FRET efficiency of the three systems calculated via the acceptor sensitation and donor quenching. The values for the calculation of the FRET efficiency can be found in Table 3 
Table 3 Calculation of the FRET efficiency according to Eqs. 3 and 4

\begin{tabular}{llllllc}
\hline Sample & $I_{D}$ (a.u.) & $I_{D} A$ (a.u.) & $I_{A}$ (a.u.) & $I_{A} D$ (a.u.) & DQ (\%) & AS $(\%)$ \\
\hline Mixture & 30,891 & 11,415 & 5468 & 39,337 & 63 & 12.4 \\
Bond & 46,750 & 32,693 & 3534 & 17,236 & 30 & 7.8 \\
Crossing & 16,239 & 8868 & 2179 & 3459 & 45 & 1.2 \\
\hline
\end{tabular}

The areas under the peaks were determined by fitting Gaussian peaks. The exact procedures and the determination of the ratio $\epsilon_{A} / \epsilon_{D}$ (0.02) can be found in the supplementary information (Figure $\mathrm{S} 1$ ). $\mathrm{I}_{D} \ldots$ Intensity of the donor peak in the absence of acceptor $\mathrm{I}_{D A} \ldots$ Intensity of the donor peak in the presence of acceptor $\mathrm{I}_{A} \ldots$ Intensity of the acceptor peak in the absence of donor $\mathrm{I}_{A D} \ldots$ Intensity of the acceptor peak in the presence of donor DQ... Donor quenching according to Eq. 3 AS. . Acceptor sensitation according to Eq. 4

pHema films dyed with either FTSC or DCCH were produced. In a next step these films were subjected to the bonding procedure described in the experimental section. Similar to the fibers we produced bonds and crossings as a reference. If the system works the bonds should exhibit a significantly higher FRET signal than the crossings but should not exceed the mixture as this is the maximum signal that one should be able to achieve at a constant concentration of dyes in the films. In Fig. 11b one can see the results for the bonded films. As expected, the FRET signal is stronger than the FRET response in Fig. 11c but weaker than in the case of the mixed film. In Fig. 11c one can see the spectroscopy measurement of the crossed but not bonded thin films. This measurement serves as a reference for unbonded surfaces as in this case the dyes are not capable of interacting with each other. This result is a good example on why it is not sufficient and reliable to only use donor quenching as an indication for FRET. As can be seen in Fig. 11d the result from the donor quenching indicates a higher FRET signal for the crossings while the FRET efficiency from the acceptor sensitation correctly shows a lower signal than the bonded films. The reason for the lower reliability of donor quenching is because a reduction in the donor signal can come from other reasons than energy transfer such as environmental quenching (Meer 2013). Here the lower donor signal is likely due to the gap of air between the thin films that only exists in the crossed but unbonded samples. Although measured with the same instrument settings, the overall intensity of the crossed films is much lower than in the within and bonded samples as seen in Fig. 11. Therefore, it is necessary to use the acceptor sensitation as a more reliable measure for FRET.

One reason why the pHema system works could be the increased concentration of dye molecules in the films compared to the fibers. The concentrations of dye molecules in the pHema film can be seen in Table 1 . The concentration of the fibers was estimated by measuring the intensity of the dyed fibers and comparing it to the thin films with a known concentration. Using the measured quantum yield and thickness of the systems the concentrations in Table 2 were calculated. As can be seen the concentrations differs by a factor of approx. 15. An increased concentration means that the potential of dyes to be much closer increases and also the amount of interacting dyes increases. Both leads to an increase in the FRET efficiency.

Another reason could be the increased signal to noise ratio. First the films $(1.5 \mu \mathrm{m})$ are thinner than the paper fibers $(10 \mu \mathrm{m})$. But also due to the temperature initiated bonding procedure very close to the melting point of pHema it is likely that the dyes within the films were diffusing into the other film. Both the decreased thickness and the diffusion of the dyes lead to an increased signal to noise ratio.

In summary the experiments with the pHema thin films permit two important conclusions. With the film with the mixed dyes it is demonstrated that the dyes DCCH and FTSC provide a working FRET system. The experiments with the bonded and unbonded films demonstrate that this FRET system, while it has not been able to indicate bonding between fiber surfaces, is able to indicate the bonding between the pHema thin films.

FRET in pHema model films using fluorescence wide field microscopy

In this final experiment we wanted to see if it is possible to replicate the FRET results obtained by the photo spectroscopy setup using widefield fluorescence microscopy. The reason why this is more difficult is 
that for widefield microscopy the camera only provides intensity data instead of the full spectral information. Of course on the other hand one gains spatial information on where in the sample the signal stems from and one might even be able to detect local intensity differences and differentiate between the strength of interaction. Therefore, we again produced pHema bonds ( 11 bonds) and crossings (10 crossings) and also investigated a film with a mixture of dyes in it. Figure 12 shows the same kind of samples as investigated by the spectrofluorometer. In Fig. 13 the average values of the $\mathrm{N}_{\text {FRET }}$ intensity can be seen and show the same trend as in Fig. 11d. The $\mathrm{N}_{\text {FRET }}$ of the mixture is the highest as there, almost all the DCCH and FTSC molecules interact due to the homogeneous distribution of molecules. But also the bonds exhibit a 3 times higher FRET signal than the crossings as seen in Fig. 13 (subfigure). These results highlight that it is possible to measure a FRET signal between substrates using widefield microscopy.
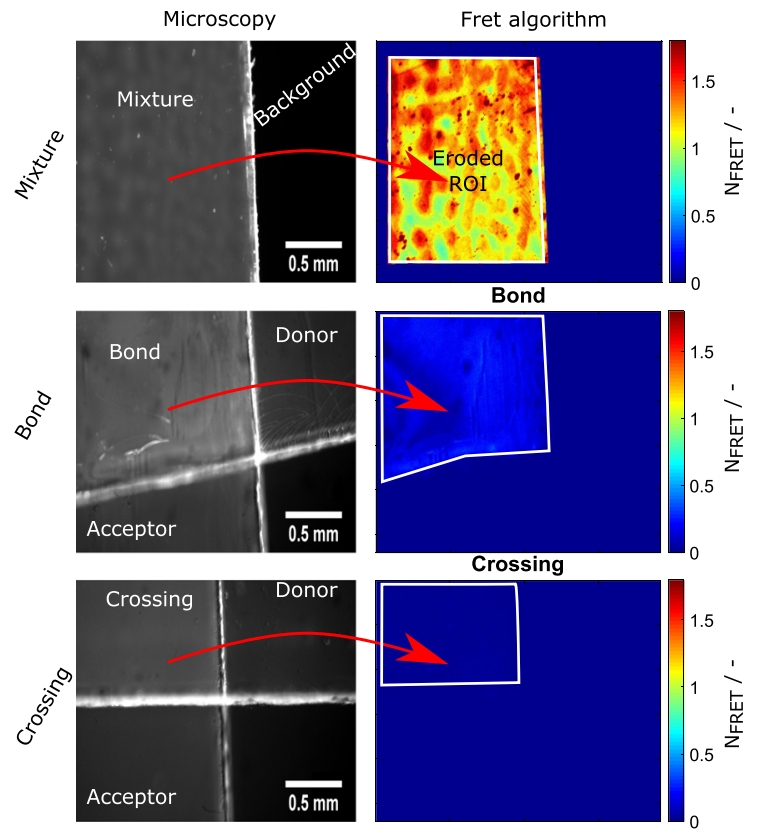

Fig. 12 Comparison of the FRET measurements between 3 types of systems using fluorescence microscopy. a Strong FRET signal stemming from the interaction of dyes mixed (Mixture) together within one film. Here also the image erosion technique used to analyze the images can be seen. b Weaker FRET signal coming from two separate films bonded (bond) together. c No FRET signal in the case of unbonded (crossing) films

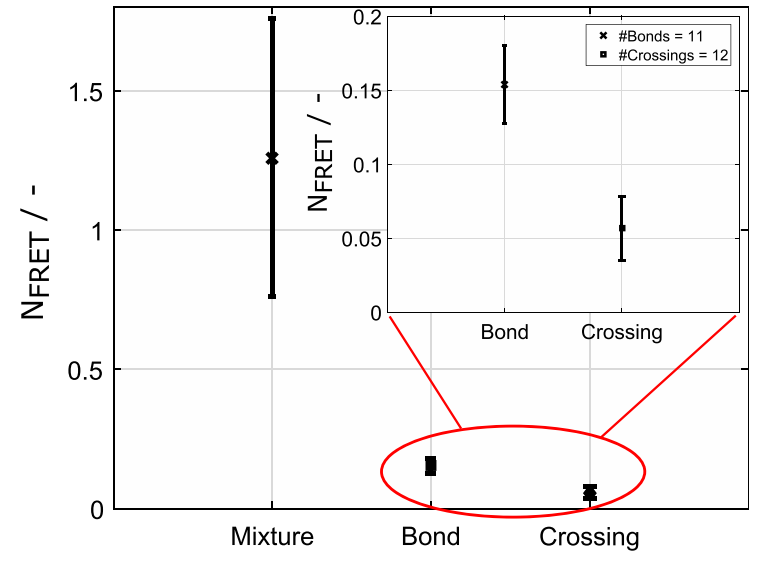

Fig. 13 Statistical analysis of the thin film models seen in Fig. 12. As expected the mixture shows the highest FRET response. The signal from bonds is about 3 times larger than from crossings. Error bars are $95 \%$ confidence limits

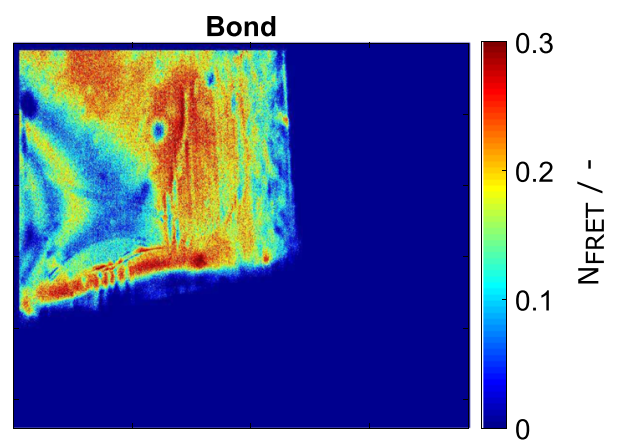

Fig. 14 Enlarged image of the $\mathrm{N}_{\text {FRET }}$ response of the pHema bond seen in Fig. 11. Here the scale was changed to emphasize the differences in the FRET signal at different positions in the film. The higher signal comes from a stronger interaction of the dyes

We also looked at the images in more detail to see if it is possible to see local variations in the FRET signals of the bonded film. Figure 14 shows the enlarged and rescaled pHema bond from Fig. 12. The higher the $\mathrm{N}_{\text {FRET }}$ value the stronger is the interaction. In the image there are regions with considerably higher FRET signals than in others. Since the dyes are not covalently linked and the bonds were formed using increased temperature it is not possible at the moment to say whether this increased signal comes from a stronger bonding (as molecules get closer the $\mathrm{N}_{\text {FRET }}$ 
increases) or interdiffusion (as more molecules interact with each other the $\mathrm{N}_{\mathrm{FRET}}$ increases). However, in either case it is a sign for local variations in bonding intensity between the surfaces.

\section{Other FRET experiments}

As briefly mentioned in the experimental Sect. 2.6 we also tried acceptor photobleaching and sensitized emission, using a CLSM. From literature we found that acceptor photobleaching promises to be a rather simple but reliable FRET experiment (Jares-Erijman and Jovin 2003) and by using a CLSM we were hoping that due to the improved microscopy setup with greater focus and sensitivity a solution to the problems stated above would be found. However also with the CSLM it was not possible to detect a FRET signal when investigating the fiber-fiber bonds.

\section{Conclusions}

In this work we investigate the possibility of measuring molecular interactions between two bonded surfaces using the effect of Förster Resonance Energy Transfer. We measured the degree of molecular contact for paper fibers and pHema model films dyed with DCCH and FTSC. In bonded paper fibers we were unable to detect a significant FRET intensity which is likely due to the poor signal to noise ratio of the system, as the bulk of the fibers is fully dyed and only the bonded surface gives a FRET signal. Thus we recommend only surface active dyeing when investigating molecular interaction between surfaces with FRET. Another reason for the poor FRET signal could be that the concentrations of dye in the paper fibers is approximately a factor of 15 smaller than in the pHema films.

Using the pHema model films we were able to validate the FRET approach. Three types of pHema systems were investigated, one that consisted of a mixture of dyes within one film, two separately dyed films subsequently bonded together and separately dyed films crossed but not bonded. Molecular contact and FRET transfer was detected by both, spectroscopy and fluorescence microscopy, in the case of mixed dyes and bonded thin films. For fluorescence microscopy, the difference between the signals was smaller due to various obstacles such as focus, bleed through or the necessary image corrections, all affecting the signal to noise ratio. Therefore, we conclude that in principle it is possible to quantify the degree of contact on the nanometer scale between two surfaces with the FRET system we have used. However, we have not been able to reproduce FRET between paper fibers as published elsewhere (Thomson et al. 2007, 2008a, b).

Acknowledgments The authors thank the Austrian Federal Ministry of Economy, Family and Youth and the Austrian National Foundation for Research, Technology and Development for their financial support. Also the industrial partners Mondi, Océ, SIG Combibloc and Kelheim Fibres are acknowledged. Furthermore this research has received funding from the EU Horizon 2020 program under Marie SklodowskaCurie Grant Agreement No. 764713, ITN Project FibreNet. Finally the authors thank Christoph Staudinger for valuable discussions and help with some of the experiments. Funding was provided by Christian Doppler Forschungsgesellschaft. Open access funding provided by Graz University of Technology.

Open Access This article is distributed under the terms of the Creative Commons Attribution 4.0 International License (http:// creativecommons.org/licenses/by/4.0/), which permits unrestricted use, distribution, and reproduction in any medium, provided you give appropriate credit to the original author(s) and the source, provide a link to the Creative Commons license, and indicate if changes were made.

\section{References}

Asunaa S, Steenberg B (1958) Beaten pulps and the fibre-tofibre bond in paper. Svensk Papperstidning 61:686-695

Broussard JA, Rappaz B, Webb DJ, Brown CM (2013) Fluorescence resonance energy transfer microscopy as demonstrated by measuring the activation of the serine/ threonine kinase Akt. Nat Protoc 8(2):265-281

Clegg RM (1992) [18] Fluorescence resonance energy transfer and nucleic acids. In: Methods in enzymology, vol 211. Elsevier, pp 353-388

Clegg RM (1995) Fluorescence resonance energy transfer. Curr Opin Biotechnol 6(1):103-110

Förster T (1948) Zwischenmolekulare Energiewanderung und Fluoreszenz. Ann Phys 437(1-2):55-75

Gordon GW, Berry G, Liang XH, Levine B, Herman B (1998) Quantitative fluorescence resonance energy transfer measurements using fluorescence microscopy. Biophys J 74(5):2702-2713

Haselton WR (1954) Gas adsorption by wood, pulp, and paper. Tappi J 37:404-412

Hirn U, Schennach R (2015) Comprehensive analysis of individual pulp fiber bonds quantifies the mechanisms of fiber bonding in paper. Sci Rep 5:10503

Hirn U, Schennach R (2017) Fiber-fiber bond formation and failure: mechanisms and analytical techniques. In: Trans. 
of the 16th Fundamental Research Symposium, Oxford, pp 839-863

Hirn U, Schennach R, Ganser C, Magnusson M, Teichert C, Östlund S (2013) The area in molecular contact in fiberfiber bonds. In: Trans. of the 15th fundamental research symposium, Cambridge, pp 201-226

Jares-Erijman EA, Jovin TM (2003) Fret imaging. Nat Biotechnol 21(11):1387

Kappel L, Hirn U, Bauer W, Gilli E, Schennach R (2010) Revisiting polarized light microscopy for fiber-fiber bond area measurement - part I: theoretical fundamentals. Nordic Pulp Paper Res J 25(1):65-70

Lindström T, Wågberg L, Larsson T (2005) On the nature of joint strength in paper-a review of dry and wet strength resins used in paper manufacturing. $\mathrm{p} 111$

Meer BWVD (2013) Förster theory. In: FRET - Förster resonance energy transfer. Wiley-Blackwell, pp 23-62

Persson BN, Ganser C, Schmied F, Teichert C, Schennach R, Gilli E, Hirn U (2012) Adhesion of cellulose fibers in paper. J Phys Condens Matter 25(4):045002

Persson BNJ (2003) On the mechanism of adhesion in biological systems. J Chem Phys 118(16):7614-7621

Persson BNJ, Albohr O, Tartaglino U, Volokitin AI, Tosatti E (2004) On the nature of surface roughness with application to contact mechanics, sealing, rubber friction and adhesion. J Phys Condens Matter 17(1):R1-R62

Sormunen T, Ketola A, Miettinen A, Parkkonen J, Retulainen E (2019) X-ray nanotomography of individual pulp fibre bonds reveals the effect of wall thickness on contact area. Sci Rep 9(1):4258

Thomson C, Lowe R, Page D, Ragauskas A (2008a) Exploring fibre-fibre interfaces via FRET and fluorescence microscopy. J Pulp Paper Sci 34(2):113-120

Thomson CI, Lowe RM, Ragauskas AJ (2007) Imaging cellulose fibre interfaces with fluorescence microscopy and resonance energy transfer. Carbohydr Polym 69(4):799-804

Thomson CI, Lowe RM, Ragauskas AJ (2008b) First characterization of the development of bleached kraft softwood pulp fiber interfaces during drying and rewetting using FRET microscopy. Holzforschung 62(4):383-388

Torgnysdotter A, Kulachenko A, Gradin P, Wågberg L (2007) The link between the fiber contact zone and the physical properties of paper: a way to control paper properties. J Compos Mater 41(13):1619-1633

Xia Z, Liu Y (2001) Reliable and global measurement of fluorescence resonance energy transfer using fluorescence microscopes. Biophys J 81(4):2395-2402

Yang C, Persson BNJ, Israelachvili J, Rosenberg K (2008) Contact mechanics with adhesion: interfacial separation and contact area. EPL 84(4):46004

Publisher's Note Springer Nature remains neutral with regard to jurisdictional claims in published maps and institutional affiliations. 